\title{
La chirurgie d'un jour et le rétablissement chez les femmes qui présentent une lésion suspecte du sein : évaluation d'une intervention infirmière psychoéducative
}

par Nicole Allard

\section{Abrégé}

L'objectif de cette étude était d'évaluer la capacité d'une intervention infirmière basée sur la théorie de l'auto-régulation, nommément l'Intervention sur le focus attentionnel, l'évaluation et la gestion des symptômes (FAEGS), d'aider les femmes atteintes d'un cancer du sein et ayant subi une chirurgie d'un jour à mieux gérer leur douleur et à réduire leur détresse émotionnelle. L'échantillon comprenait 117 patientes atteintes d'un cancer du sein et subissant une intervention chirurgicale en clinique externe à titre de premier traitement anticancéreux. Toutes les participantes ont été interviewées à trois reprises. Elles ont été randomisées dans un groupe expérimental $(n=$ 61) ou dans un groupe contrôle $(n=56)$ qui recevait les soins habituels. Les membres du groupe expérimental recevaient les interventions en deux séances, soit 3-4 jours et 10-11 jours après la chirurgie. La douleur et la détresse émotionnelle des participantes constituaient les résultats à mesurer. Les résultats après l'intervention FAEGS présentaient d'importantes différences entre les deux groupes, en post-test, dans les domaines suivants : gestion du domicile, perturbation totale de l'humeur, confusion et tension. Ces différences indiquaient que l'intervention avait réussi à atteindre certains des résultats escomptés. Des études ont montré que les interventions infirmières qui sont effectuées au tout début de la période de rétablissement d'une opération liée au cancer du sein jouent un rôle clinique important dans la réduction de la détresse émotionnelle. La théorie de l'auto-régulation pourrait très bien servir de guide dans la création de programmes d'interventions infirmières à l'intention de patients atteints d'un cancer et subissant une chirurgie d'un jour à titre de traitement primaire.

\section{Introduction}

Le cancer du sein est la forme de cancer la plus fréquente parmi les femmes de 35 à 55 ans, tant au Québec qu'au pays. Il représente $30 \%$ de tous les cas de cancer chez les femmes, et $18 \%$ des décès dus au cancer (Institut national du cancer du Canada, 2004). Pour la majorité des femmes atteintes d'un cancer du sein primaire, la chirurgie constitue le premier traitement offert. Le traitement que reçoivent la plupart des femmes comprend dorénavant une tumorectomie locale et une radiothérapie. Dans le passé, les femmes demeuraient à l'hôpital quelques jours après l'opération, pendant lesquels les infirmières fournissaient des soins postopératoires et des instructions pour l'administration d'autosoins. Traditionnellement, les femmes passaient ces quelques jours à l'hôpital pour recevoir des soins infirmiers, se rétablir physiquement et pour faciliter la gestion des drains (Bundred et coll., 1998; Kambouris, 1996).

Cependant, en raison de la récente restructuration du système de santé, la durée des séjours à l'hôpital s'est considérablement écourtée, entraînant ainsi la réduction des contacts avec les professionnels de la santé. Bien qu'elle soit apparemment sécuritaire et économique, la chirurgie d'un jour implique une responsabilité accrue pour les

Auteure pour la correspondance : Nicole Allard, inf., PhD,

Professeure, Université du Québec à Rimouski, Campus de Lévis,

55 rue du Mont-Marie, Lévis, Quebec, 833-8800 poste 3274,

1-800-463-4713 (pour les personnes de l'extérieur)

nicole_allard@uqar.qc.ca patients et leur famille sur le plan des soins postopératoires et de la gestion des symptômes, ce qui peut présenter des défis (Sladek, Swenson, Ritz et Schroeder, 1999).

\section{Recension de la documentation scientifique}

La recherche antérieure a montré que de nombreux symptômes attendus et inattendus, tels que la douleur, la perte de sensibilité dans la région du sein, la fatigue, l'anxiété et les difficultés à dormir, peuvent survenir immédiatement après la chirurgie liée au cancer du sein, peu importe le type de chirurgie (c.-à-d. mastectomie ou tumorectomie) (Baron et coll., 2002; Baron et coll., 2000; Bundred et coll., 1998; Hoskins, 1997; Johnson, Fieler, Wlasowicz, Mitchell et Jones, 1997; Maunsell, Brisson et Deschenes, 1993). Les complications entourant les différents types de chirurgie liée au cancer du sein comprennent : la douleur chronique, les hématomes, les séromes, les infections de la plaie opératoire et/ou la mobilité réduite de l'épaule (Bonnema et coll., 1998; Bundred et coll., 1998; Deo, Shukla, Goel et Kishore, 1997). La douleur après une opération liée au cancer du sein découle vraisemblablement d'une blessure chirurgicale au nerf intercostobrachial (une branche cutanée du nerf T1-2) à la suite d'une dissection axillaire (Wallace et Irving, 1997).

La fatigue peut être une préoccupation pour les personnes atteintes d'un cancer à différentes étapes de leur maladie : avant le diagnostic ou le traitement (Cimprich, 1999), pendant ou après le traitement (Irvine, Vincent, Graydon, Thompson, 1991) ou durant les étapes plus tardives de la trajectoire de la maladie (Glaus, 1993). Parmi les symptômes du cancer et ses traitements, la fatigue est celui qui est le plus courant et qui cause le plus de détresse (Blesch et coll., 1991; Irvine, Vincent, Graydon et Bubela, 1998; Wyatt et Friedman, 1998).

L'insomnie est un autre symptôme courant parmi les patients atteints de cancer. Toutefois, en raison du nombre réduit d'études empiriques sur ce problème, il est impossible de tirer des conclusions définitives sur sa prévalence (Yellen et Dyonzak, 1996). Le sommeil est très sensible aux perturbations émotionnelles ou à d'autres facteurs liés à la santé, lesquels peuvent provoquer une insomnie aiguë (Morin, 1993). Les conséquences de l'insomnie comprennent les perturbations de l'humeur, la fatigue, la réduction de la capacité fonctionnelle, la gêne en situation sociale, la détresse prononcée, la diminution des capacités cognitives et du fonctionnement quotidien et un impact considérable sur la qualité de vie (Morin, 1993).

Des conclusions cliniques et empiriques indiquent que la phase du diagnostic de cancer du sein constitue un moment extrêmement stressant pour les femmes qui est caractérisé par une anxiété prononcée et des difficultés à prendre des décisions (Northouse, 1989). Après la chirurgie, de nombreuses femmes s'inquiètent de la présence de cellules cancéreuses résiduelles, et elles veulent commencer un traitement adjuvant dès que possible (Iocolano, 1994; McIlmoyl, 1998). Durant la phase de convalescence, les femmes doivent s'adapter à des changements dans les rôles familiaux, composer avec leurs peurs relatives au diagnostic final et à la récurrence et apprendre à équilibrer les besoins des membres de leur famille touchés par cette expérience (Northouse, 1992).

La capacité fonctionnelle est définie comme la capacité d'effectuer les tâches quotidiennes habituelles. Ganz, Schag, Polinsky, Heinrich, Flack (1987) ont observé que dans le premier mois suivant la chirurgie liée au cancer du sein, les femmes $(n=50)$ qui avaient subi une 
tumorectomie $(40 \%)$ ou une mastectomie $(60 \%)$ signalaient une large gamme de problèmes physiques et de perturbations de la capacité fonctionnelle. Ces problèmes comprenaient des difficultés à soulever des charges, une mobilité réduite dans les extrémités supérieures et des difficultés à effectuer les tâches ménagères. Schag et ses collègues (1993) ont également observé qu'un mois après la chirurgie, les femmes atteintes d'un cancer du sein $(n=227)$ présentaient des baisses prononcées de leur capacité de réaliser leurs activités quotidiennes. Dans un échantillon de 117 femmes interviewées huit semaines après l'obtention du congé de l'hôpital, Bochenek (1996) rapportait que les limitations fonctionnelles les plus fréquentes étaient liées aux activités vigoureuses $(76,3 \%)$ telles que le fait de soulever des charges importantes ou la participation à des sports très physiques.

Les symptômes peuvent empêcher les femmes de maintenir leurs niveaux d'activité antérieurs, réduire leur intérêt pour les activités sociales et nuire à leur qualité de vie (Baron et coll., 2000; Mock et coll., 1997; Polinski, 1994). Les symptômes peuvent également avoir un effet considérable sur le fonctionnement. À partir de mesures obtenues au moyen de la MOS SF-36, Abent (1998) a observé que le fonctionnement physique $(r=-0,36, p=0,00)$ et le fonctionnement social $(r=-0,30, p=$ $0,00)$ affichaient une corrélation négative avec l'expérience de la fatigue $(\mathrm{n}=44)$. De plus, la douleur peut empêcher les femmes d'effectuer certaines activités de la vie quotidienne comme se peigner les cheveux ou attacher un soutien-gorge (Wyatt et Friedman, 1998).

\section{Cadre conceptuel : La théorie de l'auto-régulation}

La théorie de l'auto-régulation, rebaptisée théorie de l'autorégulation de l'adaptation à la maladie (Johnson, 1999), se base sur le traitement de l'information pour expliquer la façon dont les patients composent avec une expérience de santé stressante. La théorie de l'auto-régulation représente un cadre d'adaptation qui comprend deux voies fondamentales. Ces voies visent les fonctions spécifiques de l'adaptation (Lazarus et Folkman, 1984) : d'une part, la régulation de la réponse fonctionnelle et l'atteinte des objectifs fonctionnels (adaptation axée sur la résolution de problèmes) et, d'autre part, la régulation de la réponse émotionnelle et l'atteinte des objectifs émotionnels (adaptation émotionnelle) (Johnson, 1999).

Selon la théorie de l'auto-régulation, de l'information objective et concrète sur un événement de soins de santé peut influencer l'interprétation de l'expérience (Johnson, 1999). Les symptômes sont caractérisés comme des manifestations concrètes de la maladie, qui sont des composantes sensorielles du traitement cognitif des symptômes par l'individu. Johnson, Fieler, Wlasowicz, Mitchell et Jones, (1997) définissent l'adaptation comme les efforts fournis pour composer avec une expérience stressante, et on déduit que l'attention joue un rôle important dans le processus de l'adaptation. Le fait de déplacer le foyer d'attention des réponses émotionnelles à des aspects objectifs, concrets et fonctionnels de l'expérience des symptômes permet d'interpréter cette expérience en fonction de critères objectifs, ce qui favorise une adaptation axée sur l'atteinte de résultats fonctionnels (Johnson, 1999).

\section{Questions de recherche}

Les questions de recherche abordées dans le cadre de cette étude étaient les suivantes :

1. Quels sont les effets de l'intervention sur le focus attentionnel, l'évaluation et la gestion des symptômes (FAEGS) sur l'expérience des symptômes (douleur, fatigue et insomnie) de femmes ayant subi, en clinique externe, une chirurgie liée au cancer du sein (diagnostiqué ou présumé)?

2. Quels sont les effets de l'intervention FAEGS sur le niveau de fonctionnement déclaré par des femmes ayant subi, en clinique externe, une chirurgie liée au cancer du sein (diagnostiqué ou présumé)?

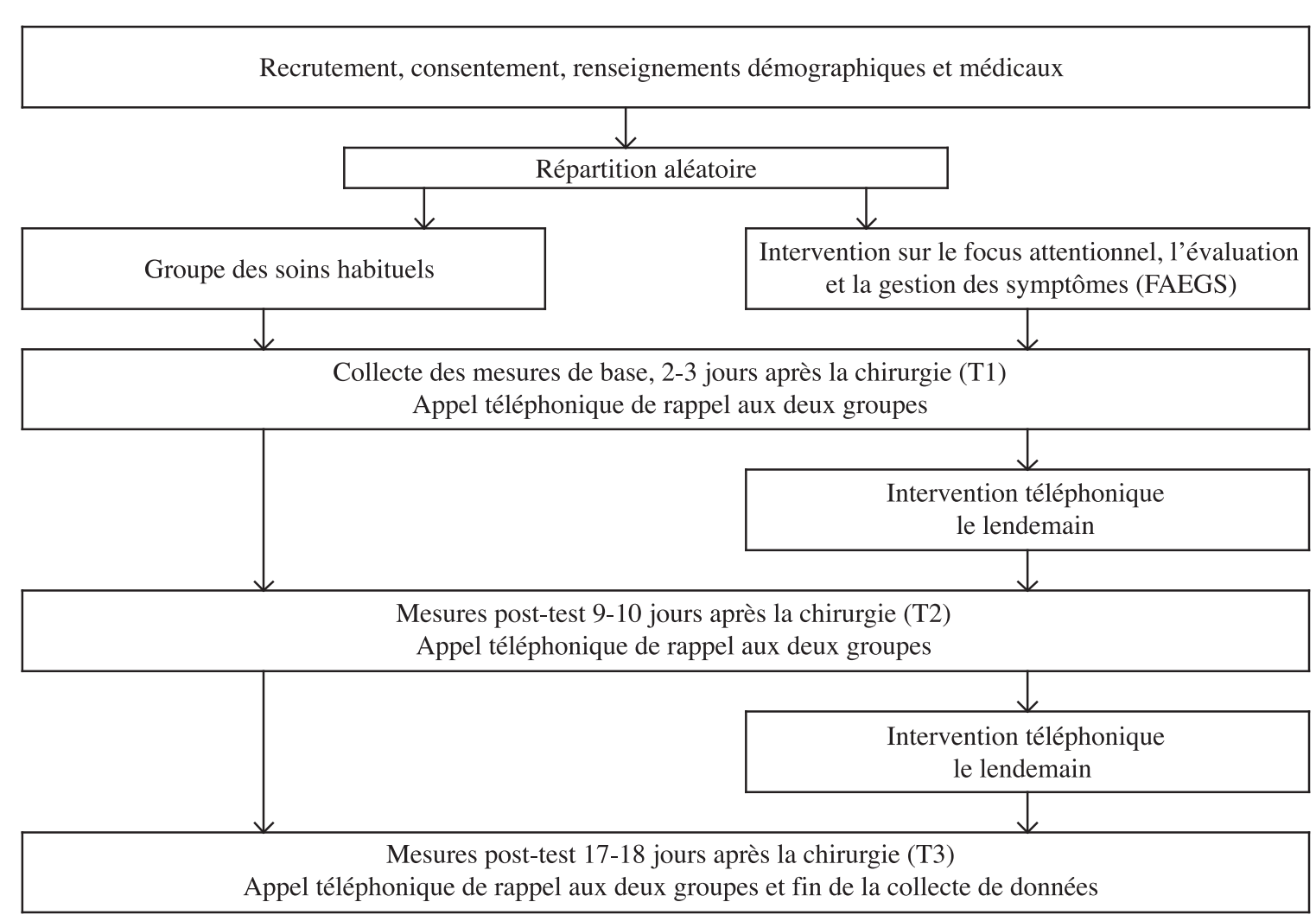

Figure 1. 
3. Quels sont les effets de l'intervention FAEGS sur le niveau de détresse émotionnelle déclaré par des femmes ayant subi, en clinique externe, une chirurgie liée au cancer du sein (diagnostiqué ou présumé)?

\section{Méthodologie \\ Échantillon}

La population cible comprenait des femmes du Québec qui venaient d'être diagnostiquées d'un cancer du sein et qui devaient subir une tumorectomie dans le cadre d'une chirurgie d'un jour. Pour participer à l'étude, les femmes devaient : 1) avoir un cancer du sein primaire ou une lésion présumée en ce même site; 2) pouvoir parler, comprendre, lire et écrire la langue; 3 ) avoir plus de 18 ans; 4) ne pas avoir de trouble d'audition; 5) avoir le téléphone à domicile. Nous avons exclu de l'étude les femmes qui avaient déjà eu une expérience de cancer ou de graves troubles psychiatriques tels qu'une psychose. Nous avons inclus dans l'échantillon des femmes n'ayant pas reçu de diagnostic final de cancer avant la chirurgie, parce que la recherche a montré que ces femmes affichent des niveaux de détresse psychologique semblables (Woodward et Webb, 2001). Nous nous attendions à ce que l'intervention FAEGS ait un effet d'une ampleur modérée sur les résultats. L'échantillon total devait comprendre 128 femmes (64 par groupe) si nous souhaitions obtenir une puissance de 0,80 et une probabilité d'erreur de type I de 0,05 (Cohen, 1992). Toutefois, en raison de difficultés de recrutement, l'échantillon final ne comprenait que 117 femmes.

\section{Établissements de santé}

L'échantillon de convenance regroupait des femmes de quatre (4) centres régionaux, dont un comprenait deux sites. Ainsi, l'analyse portait sur cinq (5) sites différents. Les quatre centres étaient situés dans différentes régions urbaines et rurales du Québec, au Canada. La plupart des femmes recevaient des soins dans le site double $4 \mathrm{a}$ et $4 \mathrm{~b}$ $(\mathrm{n}=55,47 \%)$, suivis du site $1(\mathrm{n}=25,21,4 \%)$, du site $3(\mathrm{n}=24$, $20,5 \%)$ et finalement, du site $2(\mathrm{n}=13,11,1 \%)$.

\section{Soins habituels}

Les membres du groupe contrôle ont reçu les soins habituels. Au Québec, cela comprend généralement la prestation d'un enseignement par les infirmières à toutes les femmes avant la chirurgie liée au cancer du sein et immédiatement avant le congé de l'hôpital. Au cours des 24 heures suivant leur congé, les femmes ont reçu un appel téléphonique de suivi de la part d'une infirmière de chevet et d'une infirmière en santé communautaire du Québec. Les infirmières s'enquéraient de l'état des femmes et leur demandaient si leur chirurgien leur avait prescrit des soins à domicile. Les infirmières en santé communautaire n'avaient pas de formation spécialisée en soins postopératoires liés aux chirurgies du sein. Les femmes du centre urbain devaient se rendre au centre de santé communautaire si elles avaient besoin de soins infirmiers. En plus de recevoir les soins habituels, les membres du groupe contrôle devaient se soumettre à une nouvelle évaluation des résultats à T1, T2 et T3, comme prévu dans le plan de l'étude (Figure 1). Elles avaient accès aux mêmes services d'aiguillage que le groupe expérimental si elles exprimaient le besoin de recevoir un soutien additionnel.

\section{L'intervention sur le focus attentionnel, \\ l'évaluation et la gestion des symptômes (FAEGS)}

L'intervention FAEGS (Figure 2) a été mise au point par l'auteure (Allard, 2001) à partir du travail de Dodd (1994) visant à encourager les patientes à centrer leur attention sur les symptômes qui se présentent après la chirurgie liée au cancer du sein, sur les décisions qu'elles prennent en vue de soulager ou de gérer les symptômes (régulation de leur réponse fonctionnelle et gestion des symptômes) et sur l'atteinte d'un bien-être physique et émotionnel (résultats fonctionnels et émotionnels). L'intervention FAEGS a été créée pour aider les femmes à diriger leur attention sur la voie fonctionnelle de l'adaptation. La recherche sur l'attention vise à déterminer si une personne concentre son attention sur les sources de stress, comme les aspects objectifs de l'événement, ou sur ses réactions émotionnelles à ces mêmes sources (Lamontagne, Johnson, Hepworth et Johnson, 1997). Ce type de travail vise à 1) aider les femmes à recentrer leur attention sur ce qu'elles peuvent faire pour composer avec la situation et l'anxiété et pour maintenir leurs activités quotidiennes; 2) accroître leur confort affectif par le biais d'une réponse de rétroaction (Cote et Pepler, 2002). Comme nous l'avons mentionné plus haut, l'intervenante téléphonait chaque femme une fois par semaine pendant deux semaines, pour un total de deux séances d'intervention. Au moyen d'un guide d'entrevue et d'une feuille de suivi (Allard, 2005), les symptômes de chaque femme ont été évalués, en lui

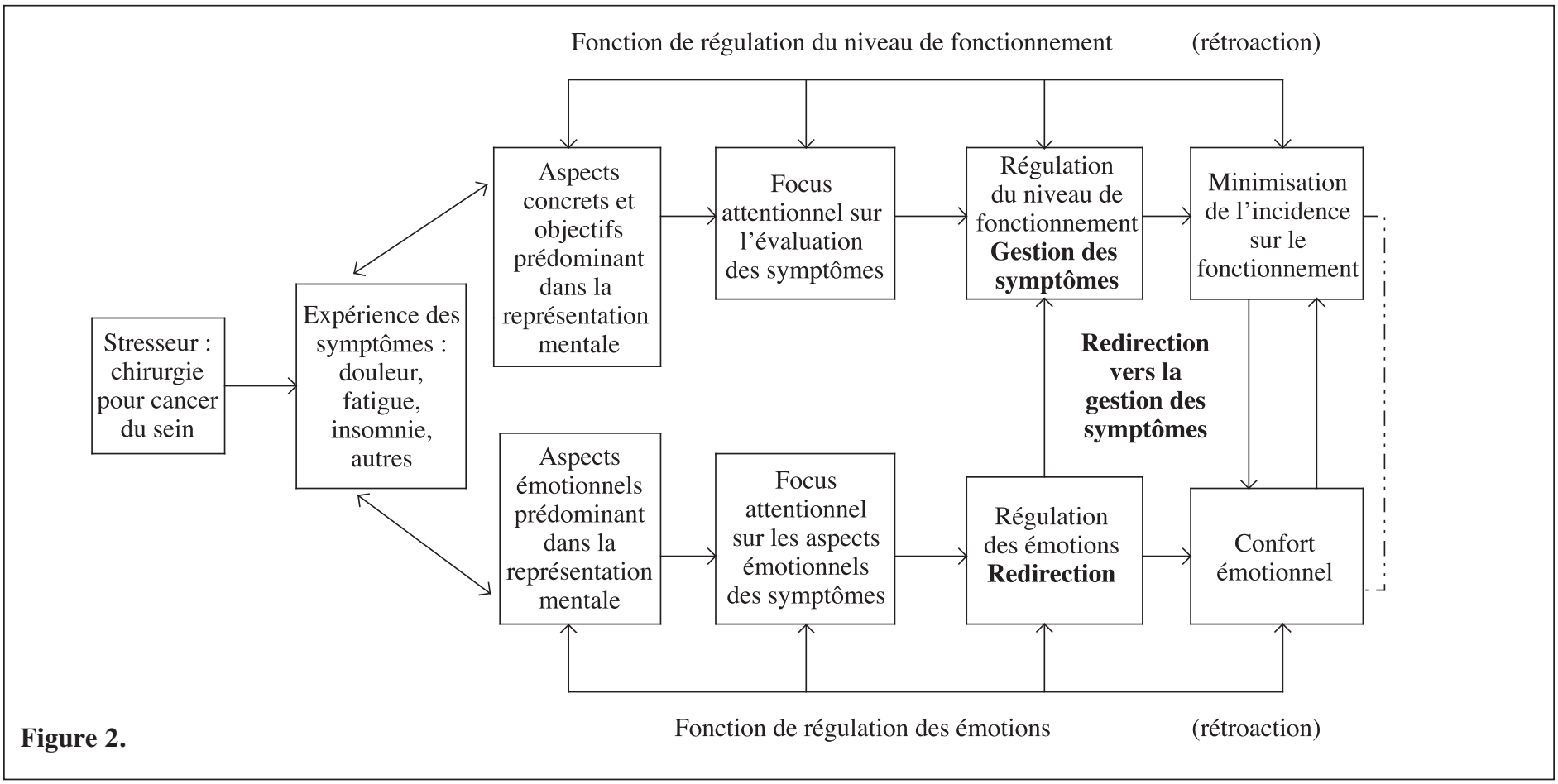


demandant de nommer et de décrire tous ses symptômes au moyen de termes objectifs et concrets. Les mesures prises par les femmes pour gérer chaque symptôme et l'efficacité de ces actions dans le soulagement des symptômes ont également été examinées. L'instrument employé pour cette évaluation était une échelle de Likert de cinq points, dans laquelle 1 signifiait «non efficace » et 5 signifiait « très efficace ». L'intervenante encourageait les femmes à continuer de prendre les mesures qu'elles estimaient efficaces dans la gestion de leurs symptômes. Si les mesures étaient inefficaces, les femmes étaient invitées à trouver d'autres mesures potentiellement efficaces. Sur demande, l'intervenante suggérait des stratégies d'autosoins nouvelles ou additionnelles. Durant l'entretien téléphonique, l'intervenante reconnaissait tous les sentiments et émotions exprimés par les femmes. Elle a soigneusement documenté et analysé les autres symptômes ou préoccupations au moyen d'une analyse du contenu. La durée des entretiens téléphoniques dépendait du nombre de symptômes éprouvés ou des autres préoccupations soulevées par les femmes.

\section{Conception de l'étude de recherche}

Un essai clinique aléatoire prospectif a été réalisé avec mesures répétées pour déterminer l'effet de l'intervention FAEGS sur l'expérience des symptômes, la détresse émotionnelle et la capacité fonctionnelle. Dans chaque site, les participantes ont été randomisées soit au groupe contrôle, soit au groupe expérimental. Le type de chirurgie (avec ou sans dissection axillaire) a été introduit en tant que variable de blocage, en raison de son effet potentiel sur la variable des résultats. Les membres du groupe contrôle ont reçu les soins habituels seulement (c.-à-d. un appel de suivi), tandis que les membres du groupe expérimental ont reçu les soins habituels ainsi que l'intervention FAEGS.

\section{Procédure}

La permission de réaliser l'étude dans les sites participants a été obtenue auprès de chacun des comités d'éthique de la recherche. Toutes les patientes disposées à participer ont donné aux infirmières de chevet la permission orale de communiquer leur numéro de téléphone à l'assistante de recherche (AR). Afin de recueillir toutes les données, l'AR a utilisé un guide élaboré d'avance. On a demandé à chaque participante volontaire de signer et poster, dans une enveloppe de retour préaffranchie, un exemplaire du formulaire de consentement destinée à la chercheuse afin d'être admissible à l'étude. En vue de réduire au minimum l'influence potentielle du site, la randomisation au groupe contrôle ou au groupe expérimental a été faite pour chaque site séparément au moyen d'un tableau de nombres randomisés (Polit et Beck, 2004). Une méthode d'échantillonnage stratifié a été employée dans laquelle l'échantillon total avait été divisé en deux catégories, à savoir les femmes subissant une biopsie des ganglions lymphatiques axillaires et celles ne subissant pas une telle intervention. Puis, les femmes de ces deux catégories ont été randomisée aux groupes contrôle et expérimental. Le groupe expérimental a ainsi accueilli 50 femmes subissant une dissection axillaire et 11 ne subissant pas cette intervention, tandis que le groupe contrôle accueillait 46 femmes de la première catégorie et 10 de la seconde. Aux mêmes trois points dans le temps, l'AR a ensuite pris des mesures auprès des groupes expérimental et contrôle.

\section{Analyse des données}

Des mesures répétées de la variance (ANOVA) ont été effectuées avec quatre variables indépendantes : le groupe expérimental (deux niveaux), le site (quatre niveaux), la dissection axillaire (deux niveaux) et le temps (trois niveaux). Des analyses de la covariance ont été utilisées lorsque les mesures de base effectuées avant l'intervention FAEGS présentaient des différences d'un groupe à l'autre et d'un site à l'autre. La supposition d'égalité de la variance des mesures prises aux trois différents moments ne s'est pas avérée dans le cas de plusieurs variables. La variabilité dans les degrés de liberté se devait à la correction de la borne inférieure utilisée pour compenser pour la violation du postulat de sphéricité.

\section{Résultats}

Au cours de la période de collecte de données qui s'est étendue sur deux ans, 182 femmes ont été aiguillées vers l'étude. Vingt-sept $(15 \%)$ d'entre elles ne satisfaisaient pas aux critères d'admissibilité de l'étude, trente et une (17\%) ont refusé de participer et sept $(4 \%)$ se sont retirées de l'étude après avoir signé le formulaire de consentement. Au total, 64,28 \% des femmes approchées ont pris part à l'étude. Les principaux facteurs de non-admissibilité étaient une expérience de cancer antérieure et le fait d'avoir reçu des traitements de chimiothérapie avant la chirurgie. Les raisons données par les femmes qui refusaient de participer comprenaient l'anxiété élevée, la fatigue, la difficulté à comprendre les questionnaires et le temps requis. L'échantillon final comprenait 117 femmes. Soixante et une femmes ont été assignées au groupe FAEGS (expérimental) et 56 au groupe de soins habituels (contrôle). La durée moyenne de chaque entretien téléphonique était de 30 minutes. La durée totale de l'intervention (c.-à-d. les deux séances) allait de 2 à 112 minutes.

\begin{tabular}{|c|c|c|c|}
\hline & Instruments & Domaines & $\begin{array}{l}\text { Plage } \\
\text { des scores }\end{array}$ \\
\hline \multirow[t]{3}{*}{$\begin{array}{l}\text { Vécu des } \\
\text { symptômes }\end{array}$} & $\begin{array}{l}\text { Multidimensional } \\
\text { Fatigue Inventory } \\
\text { (MFI) (Smets) } \\
\text { (1995) Version } \\
\text { française de Fillion } \\
\text { et coll. (2003) } \\
\text { (Inventaire } \\
\text { multidimensionnel } \\
\text { de la fatigue - } \\
\text { IMF) }\end{array}$ & $\begin{array}{l}\text { Fatigue : } \\
\text { Fatigue globale } \\
\text { Fatigue physique } \\
\text { Fatigue mentale } \\
\text { Activités réduites } \\
\text { Motivation } \\
\text { réduite }\end{array}$ & $\begin{array}{l}\text { 4-20 pour } \\
\text { chaque } \\
\text { sous- } \\
\text { échelle }\end{array}$ \\
\hline & $\begin{array}{l}\text { Version française } \\
\text { abrégée du } \\
\text { questionnaire sur } \\
\text { la douleur McGill } \\
\text { d'après Melzack } \\
\text { (1987) }\end{array}$ & $\begin{array}{l}\text { Intensité actuelle } \\
\text { de la douleur } \\
\text { Douleur } \\
\text { sensorielle } \\
\text { Douleur affective } \\
\text { Douleur globale }\end{array}$ & $\begin{array}{l}0-5 \\
0-33.87 \\
0-23.22 \\
0-57.09\end{array}$ \\
\hline & $\begin{array}{l}\text { Index de sévérité } \\
\text { de l'insomnie de } \\
\text { Morinet coll. } \\
\text { (1993) Version } \\
\text { française de Morin }\end{array}$ & Insomnie & $0-28$ \\
\hline $\begin{array}{l}\text { Capacité } \\
\text { fonctionnelle }\end{array}$ & $\begin{array}{l}\text { Symptom Impact } \\
\text { Profile (1976) } \\
\text { Sous-échelles } \\
\text { traduites en } \\
\text { français par Allard } \\
\text { et Anctil (2000) }\end{array}$ & $\begin{array}{l}\text { Gestion du } \\
\text { domicile } \\
\text { Loisirs et } \\
\text { passe-temps }\end{array}$ & $\begin{array}{l}0-100 \\
\text { pour } \\
\text { chaque } \\
\text { sous- } \\
\text { échelle }\end{array}$ \\
\hline $\begin{array}{l}\text { Détresse } \\
\text { émotionnelle }\end{array}$ & $\begin{array}{l}\text { Sous-échelles et } \\
\text { score total associé } \\
\text { du Profile of } \\
\text { Mood State (1971) } \\
\text { Version française } \\
\text { de Fillion et coll. } \\
\text { (1999) Mesures } \\
\text { des réactions } \\
\text { émotives }\end{array}$ & $\begin{array}{l}\text { Colère } \\
\text { Dépression } \\
\text { Anxiété } \\
\text { Confusion } \\
\text { Perturbation } \\
\text { totale de } \\
\text { l'humeur }\end{array}$ & $\begin{array}{l}7-35 \\
8-40 \\
7-35 \\
5-25 \\
27-135\end{array}$ \\
\hline
\end{tabular}




\section{Caractéristiques de l'échantillon avant l'intervention}

L'âge moyen de l'échantillon total était de 53,6 ans (écart-type $10,17)$. Le groupe d'âge le plus important était celui de 50-59 ans $(31,1 \%)$, suivi du groupe de 60-69 ans $(26,2 \%)$. Environ $37 \%$ des femmes avaient terminé le secondaire, et $46 \%$ d'entre elles étaient mariées. Elles avaient des occupations variées. Ainsi, certaines étaient des femmes au foyer $(21,7 \%)$, tandis que les autres étaient professionnelles $(20,9 \%)$, à la retraite $(20 \%)$ ou avaient une situation autre $(37,4 \%)$. Il n'y avait pas de corrélation statistiquement significative entre les données sociodémographiques et les variables des résultats.

\section{Données médicales}

La plupart des femmes présentaient un cancer de stade I $(39,7 \%)$ ou de stade IIA $(25,0 \%)$. Neuf femmes ont reçu un diagnostic final de tumeur bénigne et ont subi une dissection des ganglions axillaires. Le type de chirurgie le plus fréquent était la tumorectomie avec dissection axillaire $(74,4 \%)$. Beaucoup de femmes $(26,5 \%)$ se sont fait enlever de 11 à 15 ganglions lymphatiques, suivies d'une autre tranche de $23,9 \%$ qui se sont fait enlever de 6 à 10 ganglions. La majorité des femmes $(63,2 \%)$ portaient un dispositif de drainage après la chirurgie. La plupart des femmes avaient atteint la ménopause $(59,8 \%)$.

\section{Première question de recherche \\ Fatigue et insomnie}

Dans l'ensemble, des tendances statistiques indiquaient que l'intervention FAEGS avait des effets positifs sur la fatigue physique et la perte de motivation - selon les sous-échelles de l'Inventaire multidimensionnel de la fatigue (IMF) - mais aucun effet sur les autres symptômes mesurés dans le cadre de l'étude. La fatigue et l'insomnie ne semblaient pas être très préoccupantes pour les participantes, puisque peu de femmes ont demandé d'en parler lors des séances d'intervention.

\section{Douleur}

La douleur, l'engourdissement et la sensibilité du sein et du bras constituaient des problèmes pour de nombreuses femmes du groupe expérimental. Étant donné la prévalence de la douleur dans cet

\section{Tableau 2 : Incidence des symptômes déclarés par les membres du groupe expérimental}

\begin{tabular}{|l|l|l|}
\hline $\begin{array}{l}\text { Symptômes déclarés } \\
(\mathrm{N}=61)\end{array}$ & $\begin{array}{l}\text { Séance } \\
\text { d'intervention 1 }\end{array}$ & $\begin{array}{l}\text { Séance } \\
\text { d'intervention 2 }\end{array}$ \\
\hline $\begin{array}{l}\text { Engourdissement du sein } \\
\text { ou du bras }\end{array}$ & $48,3 \%$ & $48,3 \%$ \\
\hline $\begin{array}{l}\text { Maux corporels } \\
\text { (dos, jambes, hanches) }\end{array}$ & $36,7 \%$ & $23,3 \%$ \\
\hline Anxiété, tristesse & $26,7 \%$ & $25 \%$ \\
\hline $\begin{array}{l}\text { Sensibilité du sein } \\
\text { ou du bras }\end{array}$ & $26,7 \%$ & $26,7 \%$ \\
\hline Activité ou mobilité réduite & $20 \%$ & $13,3 \%$ \\
\hline $\begin{array}{l}\text { Problèmes liés à la digestion } \\
\text { (diarrhée, constipation) }\end{array}$ & $16,7 \%$ & $15 \%$ \\
\hline Étourdissement & $10 \%$ & $6,7 \%$ \\
\hline $\begin{array}{l}\text { Changement de température : } \\
\text { frissons, bouffées de chaleur }\end{array}$ & $3,3 \%$ & $10 \%$ \\
\hline Perte d'appétit & $3,3 \%$ & $1,7 \%$ \\
\hline Perte de mémoire & $3,3 \%$ & $1,7 \%$ \\
\hline Frustration, colère & $3,3 \%$ & $3,3 \%$ \\
\hline
\end{tabular}

échantillon, seuls les résultats liés à la douleur sont présentés. Dans l'ensemble, l'intervention FAEGS n'a eu d'effet statistiquement significatif sur aucune dimension de la douleur, ce qui implique qu'elle ne réduisait pas efficacement la douleur chez ce groupe de femmes. L'importante variance au sein du groupe pourrait expliquer le fait que les effets aient été non significatifs.

\section{Autres symptômes}

Les réponses des femmes aux questions ouvertes ont fait l'objet d'une analyse de contenu. Les symptômes les plus fréquemment rapportés pendant la première semaine, lors de la première séance d'intervention, comprenaient l'engourdissement du bras ou du sein, des douleurs dans différentes parties du corps telles que des maux de gorge ou de dos et une sensibilité dans le bras ou le sein. Lors de la seconde séance d'intervention, l'engourdissement du bras ou du sein demeurait le symptôme le plus fréquemment signalé, suivi de la sensibilité dans le bras ou le sein et des douleurs corporelles. L'incidence des symptômes dans le groupe expérimental était plus faible lors de la seconde séance d'intervention, à l'exception des symptômes suivants, qui n'ont pas diminué dans le temps (voir le tableau 2) : changements de température, sensibilité du bras ou du sein, étourdissement, engourdissement du sein ou du bras et frustration ou colère.

\section{Deuxième question de recherche}

L'instrument de mesure du profil de l'impact des symptômes (SIP) a été utilisé pour mesurer le niveau de fonctionnement, et des résultats sont fournis pour chaque sous-échelle du SIP. Dans l'ensemble, l'intervention a eu un effet statistiquement significatif sur la perturbation de la capacité de gérer le domicile, ce qui signifie que l'intervention a eu un effet positif sur cette dimension particulière du fonctionnement. Aucun effet significatif sur les dimensions loisirs et passe-temps n'a été relevé.

\section{Gestion du domicile}

Les scores moyens en matière de gestion du domicile pour l'échantillon complet et les deux groupes figurent dans le tableau 3. Les groupes ont affiché une variabilité intragroupe élevée pour cette variable. Le niveau de fonctionnement en gestion du domicile était statistiquement différent d'un site à l'autre avant l'intervention FAEGS $(\mathrm{F}(4,117)=3,89, \mathrm{p}=0,01)$. Les résultats de l'analyse factorielle de la variance par mesures répétées des scores (RMANOVA) obtenus avant l'intervention FAEGS et entrés en tant que covariables ont révélé un effet temporel significatif $(F(1,98)=6,85$, $\mathrm{p}=0,01)$, ce qui signifie que durant l'ensemble de l'expérience, les membres des deux groupes ont vécu une perturbation moindre de leur capacité de gérer le domicile. Un effet de groupe significatif (F $(1,98)=4,93, p=0,03)$ a été relevé, ce qui signifie que l'intervention FAEGS a contribué à réduire la perturbation de la capacité de gérer le domicile. Aucun effet interaction groupe par temps, site ou dissection axillaire n'a été constaté. Des comparaisons ultérieures au moyen de tests t pour échantillons appariés ont révélé une baisse significative du niveau de perturbation dans les scores de gestion du domicile pour les deux groupes entre T2 et T3 ( $\mathrm{t}(107)=4,84, \mathrm{p}=0,00)$, ce qui signifie que la perturbation sur la durée était moindre. Des comparaisons ultérieures entre groupes au moyen de tests $\mathrm{t}$ pour échantillons indépendants ont révélé des tendances vers l'existence d'une différence significative entre les groupes à $\mathrm{T} 2(\mathrm{t}(115)=-1,71, \mathrm{p}=$ $0,09)$ et à $\mathrm{T} 3(\mathrm{t}(114)=-1,65, \mathrm{p}=0,10)$. Le groupe expérimental présentait un score plus faible après l'intervention FAEGS et affichait un rétablissement plus rapide. Aucun effet groupe, interaction groupe par temps ou dissection axillaire significatif n'a été constaté en ce qui concerne les dimensions de loisirs et de passe-temps.

\section{Troisième question de recherche}

Les résultats sont rapportés pour le niveau global de détresse émotionnelle ainsi que pour chaque sous-échelle du Profile of Mood State (Mesures des réactions émotives). Comme le postule la théorie 
de l'auto-régulation, les résultats ont confirmé la capacité de l'intervention FAEGS de réduire les niveaux de perturbation totale de l'humeur, de tension et de confusion.

\section{Détresse émotionnelle globale}

Les scores moyens de détresse émotionnelle globale pour l'échantillon complet et les deux groupes figurent dans le tableau 4. Avant l'intervention FAEGS, le niveau de détresse émotionnelle globale était statistiquement différent d'un groupe à l'autre et d'un site à l'autre $(\mathrm{F}(4,114)=3,36, \mathrm{p}=0,01)$. Les résultats de l'analyse factorielle de la variance par mesures répétées des scores obtenus avant l'intervention FAEGS et utilisés en tant que covariables ont révélé un effet de groupe significatif $(\mathrm{F}(1,93)=3,98, \mathrm{p}=0,05)$, ce qui signifie que l'intervention FAEGS a contribué à réduire la détresse émotionnelle globale. Aucun effet temps, interaction groupe par temps, site ou dissection axillaire n'a été constaté. Des comparaisons ultérieures entre groupes au moyen de tests $\mathrm{t}$ pour échantillons indépendants ont révélé une différence significative entre le groupe expérimental et le groupe contrôle à T2 (t $(106)=-2,20, p=0,030)$ dans les scores de détresse émotionnelle. Aucune différence significative n'a été relevée à T3. Toutefois, à T3, le groupe expérimental affichait un score inférieur.

\section{Confusion}

Les scores moyens de confusion pour l'échantillon complet et les deux groupes figurent dans le tableau 5. Les résultats de l'analyse factorielle de la variance par mesures répétées (RM-ANOVA) ont révélé un effet temporel significatif $(\mathrm{F}(2,192)=5,03, \mathrm{p}=0,01)$ et un effet d'interaction groupe par temps $(\mathrm{F}(2,192)=4,37, \mathrm{p}=0,01)$. Nous avons également relevé une tendance vers un lien significatif pour ce qui est de l'effet du groupe $(\mathrm{F}(1,96)=2,71, \mathrm{p}=0,10)$. Aucun effet significatif lié au site ou à la dissection axillaire n'a été relevé. Des comparaisons ultérieures au moyen de tests $\mathrm{t}$ pour échantillons appariés ont révélé une baisse significative des scores moyens de confusion entre $\mathrm{T} 1$ et $\mathrm{T} 2(\mathrm{t}(107)=3,05 ; \mathrm{p}=0,00)$, ce qui implique que la confusion était moindre après la première séance d'intervention. Aucune différence significative n'a été relevée dans les scores moyens de confusion entre T2 et T3 chez les deux groupes. La comparaison des groupes au moyen de tests $\mathrm{t}$ pour échantillons indépendants a révélé une différence significative entre les deux groupes à T2 seulement $(\mathrm{t}(106)=-1,96, \mathrm{p}=0,05)$.

\section{Dépression}

Les résultats des analyses de la variance par mesures répétées (RM-ANOVA) ont révélé un effet temporel significatif $(\mathrm{F}(2,190)=$ $2,99, \mathrm{p}=0,05)$. Aucun effet groupe, interaction groupe par temps, site ou dissection axillaire n'a été constaté. Des comparaisons ultérieures au moyen de tests t pour échantillons appariés ont révélé une tendance vers l'existence d'un lien significatif entre T2 et T3 (t (114) = 1,81, $\mathrm{p}=0,07)$.

\section{Colère}

Les résultats des analyses de la variance par mesures répétées (RM-ANOVA) n'ont révélé aucun effet temps, interaction groupe par temps, site ou dissection axillaire.

\section{Tension}

Les scores moyens de tension pour l'échantillon complet et les deux groupes figurent dans le tableau 6. Étant donné que la tension avant l'intervention FAEGS était différente d'un groupe à l'autre et d'un site à l'autre $(\mathrm{F}(1,115)=2,70 \mathrm{p}=0,04)$, la tension à $\mathrm{T} 1$ a été traitée en tant que covariable dans l'analyse factorielle de la variance par mesures répétées (RM-ANOVA). Les résultats de cette analyse révélaient un effet de groupe significatif $(\mathrm{F}(1,94)=5,61, \mathrm{p}=0,02)$. Aucun effet d'interaction groupe par temps, site ou dissection axillaire n'a été constaté. Les femmes du groupe expérimental déclaraient un niveau de tension plus faible que celles du groupe contrôle. Toutefois, les tests t pour échantillons indépendants n'ont révélé aucune différence significative entre les deux groupes. Il se peut que cela se doive au manque de puissance lors de comparaisons multiples utilisant les tests $\mathrm{t}$. Ces comparaisons augmentent le taux d'erreur de type I (Munro, 2001).

\section{Ampleur de l'effet}

Selon l'interprétation de Cohen, l'ampleur des effets de l'intervention FAEGS variait de nulle à modérée. L'ampleur était plus importante à T2 qu'à T3, ce qui signifie que 1'intervention FAEGS avait un impact plus important sur les résultats immédiatement après la première séance. Dans la section suivante, nous ne discuterons que des résultats significatifs.

\section{Discussion et implications}

Comme présumé, l'intervention FAEGS a eu un effet statistiquement significatif sur un des volets de la capacité fonctionnelle, nommément la gestion du domicile, ainsi que sur la détresse émotionnelle globale et la confusion. Ces résultats sont particulièrement importants puisque le fonctionnement physique et la détresse émotionnelle sont des variables très importantes parmi les femmes à cette étape de la trajectoire de la maladie. L'étude a permis aux membres du groupe expérimental d'accroître leurs connaissances au sujet de leur situation de soins, ce qui a réduit leur degré de confusion et les a aidées à mieux contrôler leur état affectif et leur niveau de fonctionnement dans le cadre du schéma intellectuel qu'elles avaient établi.

\section{Symptômes}

L'étude était axée sur trois symptômes principaux : la fatigue, la douleur et l'insomnie, symptômes choisis après un examen de la documentation scientifique existante. En bref, l'intervention FAEGS n'a pas mené à une réduction de la douleur, de la fatigue et de l'insomnie chez les femmes ayant subi une chirurgie liée au cancer du sein. L'objectif de l'intervention FAEGS n'était pas tant de fournir des instructions systématiques pour l'administration d'autosoins que de promouvoir les capacités d'auto-régulation des patientes et de leur enseigner des méthodes conventionnelles et nouvelles de gestion des symptômes communs tels que la douleur, la fatigue et l'insomnie temporaires. Il se peut que les femmes ayant subi l'intervention appliquaient déjà des stratégies efficaces de gestion de ces symptômes -

\section{Tableau 3 : Scores moyens pour la gestion du domicile selon les groupes}

\begin{tabular}{|l|l|l|l|}
\hline & $\begin{array}{l}\text { Groupe } \\
\text { expérimental }\end{array}$ & $\begin{array}{l}\text { Groupe } \\
\text { contrôle }\end{array}$ & Total \\
\hline (Plage : 0-100) & $\begin{array}{l}\text { Score moyen } \\
\text { (é.-t.) }\end{array}$ & $\begin{array}{l}\text { Score moyen } \\
\text { (é.-t.) }\end{array}$ & $\begin{array}{l}\text { Score moyen } \\
\text { (é.-t.) }\end{array}$ \\
\hline Temps 1 & $45,26(25,30)$ & $45,54(30,50)$ & $45,39(27,79)$ \\
\hline Temps 2 & $18,91(18,49)$ & $25,97(25,62)$ & $22,29(22,38)$ \\
\hline Temps 3 & $12,12(15,00)$ & $17,10(17,51)$ & $14,52(16,38)$ \\
\hline
\end{tabular}

Tableau 4 : Scores moyens pour la détresse émotionnelle

\begin{tabular}{|l|l|l|l|}
\hline & $\begin{array}{l}\text { Groupe } \\
\text { expérimental }\end{array}$ & $\begin{array}{l}\text { Groupe } \\
\text { contrôle }\end{array}$ & Total \\
\hline (Étendue 27-135) & $\begin{array}{l}\text { Score moyen } \\
\text { (é.-t.) }\end{array}$ & $\begin{array}{l}\text { Score moyen } \\
\text { (é.-t.) }\end{array}$ & $\begin{array}{l}\text { Score moyen } \\
\text { (é.-t.) }\end{array}$ \\
\hline Temps 1 & $47,23(15,51)$ & $49,96(19,76)$ & $48,5(17,59)$ \\
\hline Temps 2 & $41,20(14,69)$ & $47,91(18,12)$ & $44,38(16,68)$ \\
\hline Temps 3 & $41,03(15,87)$ & $45,61(16,41)$ & $43,18(16,22)$ \\
\hline
\end{tabular}


des stratégies qu'elles avaient acquises dans le cadre de leur vécu ou de l'enseignement dispensé avant l'obtention de leur congé. Les participantes à cette étude signalaient des niveaux plutôt faibles de gravité pour leurs symptômes. En effet, les femmes indiquaient dans leurs séances d'intervention que ces symptômes ne les dérangeaient pas. Il se peut que l'application de stratégies efficaces ait mené à une bonne gestion des symptômes et, par suite, à l'absence de discussion ou de suggestions concernant des stratégies de rechange qui auraient pu s'avérer plus efficaces que celles qui étaient déjà en place.

La variabilité dans l'expérience des symptômes chez les femmes a contribué à la variabilité dans la durée des séances d'intervention. Ces dernières ont duré deux minutes avec certaines femmes et jusqu'à 112 avec d'autres. Certaines femmes avaient besoin de plus de temps pour bien aborder tous leurs besoins. Les analyses des données médicales ont révélé que les membres du groupe expérimental indiquaient dans le questionnaire médical qu'elles avaient plus de maladies au moment de l'étude ou de maladies chroniques que les femmes du groupe contrôle $(62,7 \%$ et $42,86 \%$ respectivement). Les membres du groupe expérimental déclaraient des troubles tels que l'asthme, l'hypothyroïdie, la fibromyalgie, la dépression et l'épuisement professionnel, ainsi que des troubles cardiaques tels que l'hypertension et l'arythmie. Les professionnels de la santé doivent être conscients de l'existence de groupes de symptômes et de leurs effets nuisibles synergétiques sur la morbidité parmi les femmes (Dodd, Miaskowski et Paul, 2001). Il se peut que ce facteur ait limité la capacité de l'intervention à réduire la gravité des symptômes d'intérêt. Au cours de l'étude, les femmes qui ont subi leur chirurgie dans le site 4 , où le recrutement a été le plus fort, ont reçu avant la chirurgie un livret contenant des renseignements concrets et sensoriels sur l'expérience de la chirurgie liée au cancer du sein, ce qui a possiblement créé un biais. Il se peut également que les symptômes d'intérêt dans cette étude aient été liés au stress physiologique de la chirurgie et se soient résorbés relativement rapidement au début de la période de rétablissement.

\section{Fonctionnement}

Gestion du domicile

L'intervention FAEGS a eu un effet statistiquement significatif sur la gestion du domicile, mais non sur les loisirs et les passe-temps, deux autres dimensions du fonctionnement. Bien qu'il manque des

Tableau 5 : Scores moyens pour la confusion

\begin{tabular}{|l|l|l|l|}
\hline $\begin{array}{l}\text { Étendue du } \\
\text { POMS pour la } \\
\text { confusion : 5-25 }\end{array}$ & $\begin{array}{l}\text { Groupe } \\
\text { expérimental }\end{array}$ & $\begin{array}{l}\text { Groupe } \\
\text { contrôle }\end{array}$ & Total \\
\hline & $\begin{array}{l}\text { Score moyen } \\
\text { (é.t.) }\end{array}$ & $\begin{array}{l}\text { Score moyen } \\
\text { (é.-t.) }\end{array}$ & $\begin{array}{l}\text { Score moyen } \\
\text { (é.t.) }\end{array}$ \\
\hline Temps 1 & $9,79(3,73)$ & $9,57(4,13)$ & $9,69(3,91)$ \\
\hline Temps 2 & $7,98(3,58)$ & $9,49(3,98)$ & $8,70(3,83)$ \\
\hline Temps 3 & $8,03(3,54)$ & $8,82(3,74)$ & $8,41(3,64)$ \\
\hline
\end{tabular}

Table 6 : Scores moyens pour la tension

\begin{tabular}{|l|l|l|l|}
\hline $\begin{array}{l}\text { (ÉTENDUE: } \\
7-35)\end{array}$ & $\begin{array}{l}\text { Groupe } \\
\text { expérimental }\end{array}$ & $\begin{array}{l}\text { Groupe } \\
\text { contrôle }\end{array}$ & Total \\
\hline & $\begin{array}{l}\text { Score moyen } \\
\text { (é.-t.) }\end{array}$ & $\begin{array}{l}\text { Score moyen } \\
\text { (é.-t.) }\end{array}$ & $\begin{array}{l}\text { Score moyen } \\
\text { (é.-t.) }\end{array}$ \\
\hline Temps 1 & $13,93(5,63)$ & $14,19(5,66)$ & $14,05(5,62)$ \\
\hline Temps 2 & $11,15(4,79)$ & $12,67(5,52)$ & $11,87(5,18)$ \\
\hline Temps 3 & $11,18(5,05)$ & $12,46(4,87)$ & $11,78(4,99)$ \\
\hline
\end{tabular}

scores normatifs pour en établir la signification clinique, les membres du groupe expérimental étaient plus nombreux à afficher une amélioration de leur niveau de fonctionnement en gestion du domicile que les membres du groupe contrôle, ce qui renforce encore l'impact bénéfique de l'intervention FAEGS. Durant les séances de l'intervention, l'intervenante invitait les participantes à se concentrer sur ce qu'elles pouvaient faire pour améliorer leur rétablissement et les encourageait à appliquer des stratégies d'autosoins.

\section{Détresse émotionnelle}

L'intervention FAEGS a eu un effet statistiquement significatif sur la détresse émotionnelle globale. Ce résultat appuie l'idée d'appliquer des interventions infirmières pour aider les patientes dont le niveau de détresse émotionnelle ne peut pas être réduit (p. ex. l'échantillon à l'étude). L'intervention a contribué à une réduction plus rapide de la détresse émotionnelle pendant la période postchirurgicale. Une baisse significative de la détresse émotionnelle après la chirurgie a été observée chez les deux groupes. Cela est conforme aux résultats antérieurs (Carver et coll., 1993; Hoskins, 1997; Irvine, 1996; Northouse, 1989), montrant que la détresse émotionnelle des femmes atteintes d'un cancer du sein primaire atteint son point culminant au moment du diagnostic et de la chirurgie, et se résorbe progressivement par la suite.

\section{Confusion}

La confusion est une autre dimension de la détresse émotionnelle sur laquelle l'intervention FAEGS a eu un effet statistiquement significatif. Les patientes qui ont reçu l'intervention ont déclaré ressentir moins de confusion que celles qui ne l'ont pas reçue. L'intervention renseignait les femmes au sujet des symptômes, renforçait les stratégies d'autosoins et aidait les femmes à se concentrer sur les aspects objectifs de l'expérience de soins. Cela les a aidées à clarifier le schéma intellectuel qu'elles s'en étaient faites, c'est-à-dire à comprendre et expliquer l'image mentale qu'elles avaient de leur expérience de soins, des comportements durant l'expérience et des résultats souhaités (Johnson et coll., 1997). Il se peut que cette clarification ait contribué à la réduction de la confusion.

\section{Tension}

L'effet de groupe révélé par l'analyse de la variance par mesures répétées (RM-ANOVAS) indique que l'intervention a contribué à réduire les niveaux de tension sans toutefois montrer à quel moment cet effet a été le plus efficace. Par conséquent, l'intervention FAEGS a eu un effet statistiquement significatif sur la dimension tension de la détresse émotionnelle.

En bref, l'intervention FAEGS a permis de réguler la composante émotionnelle de l'adaptation comme le décrivent Lazarus et Folkman (1984). Nous avons obtenu ce résultat en écoutant activement les femmes, en permettant un certain degré de ventilation émotionnelle et en invitant les femmes à se concentrer avant tout sur les aspects concrets et objectifs de cette expérience chirurgicale stressante, comme le recommande la théorie de l'auto-régulation de Johnson (1997). Ces résultats confirment le besoin d'offrir des interventions infirmières personnalisées axées sur le soulagement de la confusion, de la tension et de la détresse émotionnelle des patients. Ces interventions aident les patients qui vivent une détresse émotionnelle et ceux qui demandent de l'aide pour gérer leurs réactions affectives à se concentrer sur les aspects concrets et objectifs de l'expérience après un certain degré de ventilation affective (Johnson, Fieler, Jones, Wlasowicz et Mitchell, 1997). Cela semble contredire d'autres rapports qui indiquent que la ventilation émotionnelle en tant que stratégie d'adaptation pourrait être nuisible, puisqu'elle a été associée à une détresse émotionnelle accrue (Compas et coll., 1999). Cependant, l'expression d'émotions liées à un diagnostic de cancer du sein ou à une intervention chirurgicale mène à une modulation et une 
compréhension accrues des émotions et est plus susceptible d'être adaptative et considérée comme une adaptation axée sur les émotions (Johnson, 1997).

\section{Implications théoriques}

Les femmes ont eu l'occasion de discuter de leurs préoccupations et frustrations, et de leur colère, anxiété et tristesse. Cette ventilation de leurs émotions s'est très probablement avérée efficace en tant que stratégie d'adaptation axée sur les émotions. Le fait de décrire leurs symptômes au moyen de termes objectifs et concrets leur a permis de se concentrer sur l'aspect positif de la situation et d'appliquer des stratégies de résolution de problèmes. Les stratégies axées sur les émotions sont utiles pour les femmes qui jugent qu'elles doivent endurer une situation et qui souhaitent réduire ou gérer la détresse émotionnelle associée à la chirurgie d'un jour liée au cancer du sein. Le fait d'avoir redirigé leur attention des réponses émotionnelles aux aspects objectifs, concrets et fonctionnels de l'expérience des symptômes a favorisé l'adaptation et l'atteinte d'objectifs fonctionnels (Johnson, 1999). Cette technique s'est révélée être une façon efficace de centrer l'attention sur les façons de gérer la situation (Cote et Pepler, 2002). De plus, l'intervention FAEGS a été dispensée après la chirurgie, ce qui révélait un besoin d'exposer les femmes une seconde fois à l'information sensorielle concrète qu'elles avaient reçue le jour de la chirurgie, afin de leur rappeler ce à quoi elles pouvaient s'attendre et de rehausser l'efficacité de l'information (Johnson, Fuller, Endress, et Rice, 1978).

\section{Implications pour la recherche}

Les résultats de cette étude soulèvent plusieurs questions qui pourraient être abordées dans de futures études. La première question est la suivante : quelle sont la fréquence, l'intensité et la durée optimales de l'intervention FAEGS? La recherche future devrait considérer une hausse éventuelle du dosage de l'intervention FAEGS et évaluer l'impact de différentes doses sur la gestion des symptômes. Le fait de donner aux patientes des descriptions précises de ce à quoi elles peuvent s'attendre ainsi que des suggestions concernant les autosoins peut renforcer leur confiance à l'heure de prendre des décisions liées aux stratégies d'adaptation (Johnson, 1997). Nous avons besoin d'instruments capables de mesurer les symptômes et les changements si nous souhaitons déceler les effets significatifs des interventions sur les symptômes. Récemment, Baron, Fey, Borgen et Van Zee (2004) et Baron et coll. (2002) ont mis au point un instrument de mesure capable d'évaluer les sensations au niveau du sein après la chirurgie. Cet instrument

\section{Références}

Abent, L.S.E. (1998). Women's perceptions of fatigue and quality of life following breast cancer surgery. Unpublished M.S.N., Michigan State University, Michigan.

Allard, N. (2001). Attentional Focus and Symptom Management Intervention (AFSMI). Rehabilitation \& Community Care Management, 9(4), 38-40.

Allard, N. (2005). Day surgery and recovery in women with a suspicious breast lesion: Evaluation of a psychoeducational nursing intervention. University of Toronto, Toronto.

Baron, R.H., Kelvin, J.F., Bookbinder, M., Cramer, L., Borgen, P.I., \& Thaler, H.T. (2000). Patients' sensations after breast cancer surgery. A pilot study. Cancer Pract, 8(5), 215-222.

Baron, R.H., Fey, J.V., Borgen, P.I., \& Van Zee, K.J. (2004). Eighteen sensations after breast cancer surgery: a two-year comparison of sentinel lymph node biopsy and axillary lymph node dissection. Oncol Nurs Forum, 31(4), 691-698.

Baron, R.H., Fey, J.V., Raboy, S., Thaler, H.T., Borgen, P.I., Temple, L.K., et al. (2002). Eighteen sensations after breast pourrait permettre de cerner plus exactement les préoccupations des femmes relativement à leurs symptômes. Les travaux futurs devraient également explorer l'influence des caractéristiques des participantes sur les effets de l'intervention FAEGS. La recherche devrait aborder les besoins des femmes souffrant de niveaux élevés d'anxiété ou de fatigue, puisque ces deux facteurs étaient des raisons assez fréquentes de non-participation. Ces femmes ont possiblement des besoins accrus et bénéficieraient sans doute de ce genre d'intervention. Il convient donc de concevoir le processus de recrutement de façon plus soignée. Il est possible que des caractéristiques telles que l'optimisme ou l'hypervigilance réduisent les effets de l'intervention sur les résultats (Sidani et Braden, 1998). Finalement, la recherche devrait également examiner d'autres facteurs importants tels que : a) les caractéristiques des milieux de soins dans lesquels les participantes sont recrutées, par exemple le nombre d'infirmières cliniciennes qualifiées et expérimentées et b) les attitudes du personnel envers la recherche infirmière et les politiques de gestion de la douleur.

\section{Implications pour la pratique}

Les résultats de ce projet de recherche ont illustré le fait suivant : une intervention infirmière appliquée immédiatement après une chirurgie liée au cancer du sein réduit substantiellement la détresse émotionnelle et rehausse le fonctionnement quotidien. La redirection de l'attention et la concentration sur les aspects concrets et objectifs sont prometteuses pour la mise au point d'interventions infirmières novatrices. La recherche a montré que l'adaptation de l'information aux situations particulières des clients et à leur type de cancer rehausse la satisfaction à l'égard des services de santé (Dunn et coll., 1993). Au lieu d'encourager la dépendance envers les professionnels de la santé, l'intervention FAEGS donne aux femmes les moyens d'auto-réguler leur situation. Avec des mises au point, par exemple en personnalisant davantage l'information liée au vécu et à la gestion des symptômes, le protocole d'intervention pourrait être élargi de façon à appuyer les femmes qui présentent plus de besoins, et ce, pendant une plus longue période après la chirurgie. Les infirmières peuvent faire preuve d'initiative en s'assurant que les patients évaluent, gèrent et comprennent bien leurs symptômes à la suite de n'importe quel genre de chirurgie d'un jour. Au Québec, la création d'un rôle d' « infirmière pivot » pour les patients en oncologie fait déjà l'objet de discussions, d'articles scientifiques et de présentations (de Serres et Beauchesne, 2000). Les infirmières en oncologie pourraient être les facilitatrices les plus indiquées pour les interventions téléphoniques (Sandgren, McCaul, King, O’Donnell et Foreman, 2000).

cancer surgery: a comparison of sentinel lymph node biopsy and axillary lymph node dissection. Oncol Nurs Forum, 29(4), 651659.

Blesch K.S., Paice J.A., Wickham R., Harte N., Schnoor D.K., Purl S., et al.(1991). Correlates of fatigue in people with breast or lung cancer. Oncol Nurs Forum, 18(1), 81-7.

Bochenek. (1996). Female breast cancer patients' perception of pain and physical well-bieng following surgery from breast cancer. Unpublished Master of nursing science, Michigan State University, Michigan.

Bonnema, J., van Wersch, A., van Geel, A., Pruyn, J., Schmitz, P., Paul, M., et al. (1998). Medical and psychosocial effects of early discharge after surgery for breast cancer: randomised trial. British Medical Journal, 3(16), 1267-1271.

Bundred, N., Maguire, P., Reynolds, J., Grimshaw, J., Morris, J., Thomson, L., et al. (1998). Randomised controlled trial of effects of early discharge after surgery for breast cancer. British Medical Journal, 317(7168), 1275-1279. 
Carver, C.S., Pozo, C., Harris, S.D., Noriega, V., Scheier, M.F., Robinson, D.S., et al. (1993). How coping mediates the effect of optimism on distress: a study of women with early stage breast cancer. Journal of Personality and Social Psychology, 65(2), 375-390.

Cimprich, B. (1999). Pretreatment symptom distress in women newly diagnosed with breast cancer. Cancer Nurs, 22(3), 185-194; quiz 195.

Cohen, J. (1992). A power primer. Psychological Bulletin, 112(1), 155-159.

Compas, B.E., Stoll, M.F., Thomsen, A.H., Oppedisano, G., EppingJordan, J.E., \& Krag, D.N. (1999). Adjustment to breast cancer: age-related differences in coping and emotional distress. Breast Cancer Res Treat, 54(3), 195-203.

Cote, J.K., \& Pepler, C. (2002). A randomized trial of a cognitive coping intervention for acutely ill HIV-positive men. Nursing research, 51(4), 237-244.

de Serres, M., \& Beauchesne, N. (2000). L'intervenant pivot en oncologie: un rôle d'évaluation, d'information et de soutien pour le mieux-être des personnes atteintes de cancer: document de réflexion. Québec: MSSS.

Deo, S.V.S., Shukla, N.K., Goel, A.K., \& Kishore, J. (1997). Short stay surgery for breast cancer: an audit of an experience in a regional cancer centre in northern India. European Journal of Surgical Oncology, 23, 335-338.

Dodd, M.J. (1984). Measuring informational intervention for chemotherapy knowledge and self-care behavior. Research in Nursing and Health, 7, 43-50.

Dodd, M.J., Miaskowski, C., Paul, S.M. (2001). Symptom clusters and their effect on the functional status of patients with cancer. Oncol Nurs Forum, 28(3), 465-70.

Dunn, S., Butow, P., Tattersall, M., Jones, Q., Sheldon, J., Taylor, J., et al. (1993). General information tapes inhibit recall of the cancer consultation. J Clin Oncol, 11(11), 2279-2285.

Ganz, P., Schag, C., Polinsky, M., Heinrich, R., \& Flack, V. (1987). Rehabilitation needs and breast cancer: the first month after primary therapy. Breast Cancer Research and Treatment, 10, 243-253.

Glaus, A. (1993). Assessment of fatigue in cancer and non-cancer patients and in healthy individuals. Supportive Care in Cancer, 1, 305-315.

Hoskins, C.N. (1997). Breast cancer treatment-related patterns in side effects, psychological distress, and perceived health status. Oncology Nursing Forum, 24(9), 1575-1583.

Iocolano, C.F. (1994). A qualitative study of four women during the first four months after surgery for breast cancer. Unpublished PhD, New York University.

Irvine, D.M. (1996). A critical review and meta-analysis of the literature investigating psychosocial adjustment in breast cancer. Ottawa: Health Canada.

Irvine, D.M., Vincent, L., Bubela, N., Thompson, L., \& Graydon, J. (1991). A critical appraisal of the research literature investigating fatigue in the individual with cancer. Cancer Nurs, 14(4), 188199.

Irvine, D.M., Vincent, L., Graydon, J., \& Bubela, N. (1998). Fatigue in women with breast cancer receivng radiation therapy. Cancer Nurs, 21(2), 127-135.

Johnson. (1999). Self-regulation theory and coping with physical illness. Research in Nursing and Health, 22(6), 435-448. [Record as supplied by publisher].

Johnson, J. E., Fuller, S., Endress, M. P., \& Rice, V. H. (1978). Altering patients'responses to surgery: an extension and replication. Research in Nursing and Health, 1(3), 111-121.

Johnson, J.E., Fieler, V.K., Jones, L.S., Wlasowicz, G.S., \& Mitchell, M.L. (1997). Self-regulation theory: Applying theory to your practice. Pittsburgh: Oncology Nursing Press.
Johnson, J.E., Fieler, V.K., Wlasowicz, G.S., Mitchell, M.L., \& Jones, L.S. (1997). The effects of nursing care guided by self-regulation theory on coping with radiation therapy. Oncology Nursing Forum, 24(6), 1041-1050.

Kambouris, A. (1996). Physical, psychological, and economic advantages of accelerated discharge after surgical treatment for breast cancer. American Surgeon, 62(2), 123-127.

Lamontagne, L., Johnson, J.E., Hepworth, J., \& Johnson, B. (1997). Attention, coping, and activity in children undergoing orthopaedic surgery. Research in Nursing and Health, 20, 487-494.

Lazarus, R.S., \& Folkman, S. (1984). Stress, appraisal and coping. New York: Springer Publishing Company, Inc.

Maunsell, E., Brisson, J., \& Deschenes, L. (1993). Arm problems and psychological distress after surgery for breast cancer. Canadian Journal of Surgery, 36(4), 315-320.

McIlmoyl, M.L. (1998). Moving on and the nightmare is back: the two faces of uncertainty and the breast cancer journey. Unpublished Master thesis, Queen's University, Kingston, Ontario, Canada.

Mock, V., Dow, K.H., Meares, C.J., Grimm, P.M., Dienemann, J.A., Haisfield-Wolfe, M.E., et al. (1997). Effects of exercise on fatigue, physical functioning, and emotional distress during radiation therapy for breast cancer. Oncology Nursing Forum, 24(6), 991-1000.

Morin, C. (1993). Insomnia: Psychological assessment and management. New York: Guilford Press.

Munro, B.H. (2001). Statistical methods for health care research (4 ed.). Philadelphia: Lippincott.

National Cancer Institute of Canada. (2004). Canadian cancer statistics. Ottawa: Health Canada.

Northouse, L.L. (1989). The impact of breast cancer on patients and husbands. Cancer Nursing, 12(5), 276-284.

Northouse, L.L. (1992). Psychological impact of the diagnosis of breast cancer on the patient and her family. Journal of the American Medical Womens Association, 47(5), 161-164.

Polinski, M.L. (1994). Functional status of long-term breast cancer survivors: Demonstrating chronicity. Health and Social Work, 19(3), 165-173.

Polit, D.F., \& Beck, C.T. (2004). Nursing research: principles and methods (7th ed.). Philadelphia: Lippincott Williams \& Wilkins.

Sandgren, A.K., McCaul, K.D., King, B., O’Donnell, S., \& Foreman, G. (2000). Telephone therapy for patients with breast cancer. Oncol Nurs Forum, 27(4), 683-688.

Schag, C.A.C., Ganz, P.A., Polinski, M.L., Fred, C., Hirji, K., \& Petersen, L. (1993). Characteristics of women at risk for psychosocial distress in the year after breast cancer. Journal of Clinical Oncology, 11(4), 783-793.

Sidani, S., \& Braden, C.J. (1998). Evaluating Nursing Interventions: A Comprehensive Approach. Thousand Oaks, CA: Sage Publications.

Sladek M.L., Swenson K.K., Ritz L.J., Schroeder L.M. (1999). A critical pathway for patients undergoing one-day breast cancer surgery. Clinical Journal of Oncology Nursing 3, 99-106.

Wallace, \& Irving. (1997). Post-mastectomy and post-breast surgery pain. In Pain management for the practicing physician (pp. 209-216). New York: Churchill Livingstone.

Woodward, V., \& Webb, C. (2001). Women's anxieties surrounding breast disorders: a systematic review of the literature. J Adv Nurs, 33(1), 29-41.

Wyatt, G., \& Friedman, L.L. (1998). Physical and psychosocial outcomes of midlife and older women following surgery and adjuvant therapy for breast cancer. Oncology Nursing Forum, 25(4), 761-768.

Yellen, S.B., \& Dyonzak, J.V. (1996). Sleep disturbances. In S.L. Groenwald, M.H. Frogge, M. Goodman \& C.H. Yarbro (Eds.), Cancer symptom management. Sudbury, MA: Jones and Bartlett Publishers. 